\title{
Replik zum Kommentar von C. Hess
}

\section{Pierre-François Cuénoud}

Dr. med., Vizepräsident der FMH, Departementsverantwortlicher Tarife und Gesundheitsökonomie Spitalärzte

Lieber Kollege

Es freut mich, noch einmal mit der Empörung derjenigen konfrontiert zu sein, die bis 2011 bemüht waren, die allgemeine Einführung der Fallpauschalen in der Schweiz mittels eines Moratoriums hinauszuschieben. Ihre Analyse verdient selbstverständlich einige Kommentare und Richtigstellungen.

Zuallererst sprechen Sie das Thema der Transparenz an: Diese hat sich nämlich durch die Schaffung einer landesweit einheitlichen Tarifstruktur tatsächlich verbessert und so dafür gesorgt, dass die Unterschiede zwischen den Kantonen und Spitälern erkenntlich gemacht und die verzerrenden Mechanismen identifiziert werden können. Was die Kosten betrifft, so war niemals die Rede davon, diese zu reduzieren. Es ging vielmehr darum, deren Wachstum zu bremsen. Die allgemeine Senkung der Baserates, die den Spitälern das Leben schwer macht, ist dabei ein typisches Merkmal dieser Entwicklung. Seit 2012 steigen Fallzahlen und Kosten im stationären Bereich nur leicht an, und die Überwachungsmechanismen greifen: Eine offenkundige Überaktivität wird von den Versicherern nicht mehr belohnt. Unnötige Leistungen kann somit nur noch erbringen, wer dafür in anderen Bereichen Abstriche macht. Es stimmt, dass einige wenige Einrichtungen eine solche Strategie verfolgen, indem sie die schwierigen, wenig profitablen Fälle den grossen öffentlichen Spitälern überlassen.

Obwohl seit etwa zwanzig Jahren im Gesetz verankert, wurde die Qualitätsdebatte erst kürzlich angestossen. Gleichzeitig mit der Einführung der Spitalfinanzierung hat man entsprechende Indikatoren entwickelt und durchgesetzt. Die FMH stimmt mit Ihnen überein, dass diese ganz offenkundig noch unzureichend sind; es muss eine echte Kontrolle der Indikationsqualität eingeführt werden, gemäss unseren durch die Schweizerische Akademie für Qualität in der Medizin (SAQM) verbreiteten Forderungen. Was schliesslich die Konkurrenz betrifft, so hat diese im Gesundheitswesen - ganz anders als in der Industrie - eindeutig ihre Grenzen. Man muss allerdings einräumen, dass einige Spitäler jahrelang eine Vormachtstellung genossen haben und die Rationalisierungsnotwendigkeit sich nicht nur auf ihre Finanzen, sondern auch auf ihre Verfahrensweisen und ihre Behandlungsqualität positiv auswirken kann. Grundsätzlich ist es gut, dass die Ärzteschaft die Entwicklung der finanziellen Aspekte im Gesundheitswesen kritisch beobachtet. Angesichts der Tatsache, dass zahlreiche Kolleginnen und Kollegen die klinische Arbeit gegen eher administrative Tätigkeiten eintauschen, muss man sich fragen, ob unsere Funktionsweise in den Spitälern an Attraktivität eingebüsst hat und nach den Ursachen forschen. So hat zwar die Anzahl der Stellen ohne direkten Patientenkontakt in den Spitälern in den letzten Jahren zugenommen; im Vergleich zu anderen Bereichen ist der Anteil jedoch zurückgegangen: Die Zahl der Arztstellen ist am stärksten gestiegen, noch vor derjenigen der Pflegekräfte. Auch bestimmte nicht-klinische Berufe sind heutzutage unerlässlich, wie beispielsweise Informatikfachkräfte, deren Kompetenzen bei den zahlreichen Ausfällen der elektronischen Patientendossiers benötigt werden.

Allgemein muss unbedingt auch weiterhin zwischen Entwicklung und Pflege der Tarifstruktur SwissDRG einerseits und den gleichzeitig eingeführten erheblichen Veränderungen bei der Spitalfinanzierung unterschieden werden. Genau genommen beträgt das Budget für den Betrieb der SwissDRG AG rund 2,3 Millionen Franken jährlich und ist damit von den hunderten Millionen, die Sie erwähnen, weit entfernt. Ganz objektiv und ohne naiven Idealismus betrachtet, hat die allgemeine Anwendung der Fallpauschalen in unserem Land bisher keinen messbaren Einfluss gezeigt und das ethische Verhalten unserer Kolleginnen und Kollegen nicht negativ beeinflusst. Der gesamte Prozess ist somit weder ein Erfolg noch ein Fehlschlag, sondern eine langsame Entwicklung hin zu einem durchschaubaren System, das zu wirtschaftlichen Überlegungen zwingt und gleichzeitig klinischen Erfordernissen den gebührenden Vorrang einräumt. 